\title{
First-Fit Scheduling for Beaconing in Multihop Wireless Networks
}

\author{
Peng-Jun Wan, Zhu Wang, and Hongwei Du \\ Department of Computer Science \\ Illinois Institute of Technology \\ Emails: \{wan, zwang59, hongwei.du\}@iit.edu
}

\author{
Scott C.-H. Huang, and Zhiyuan Wan \\ Department of Computer Science \\ City University of Hong Kong \\ Emails: \{shuang, zhiyuwan\}@ cityu.edu.hk
}

\begin{abstract}
Beaconing is a primitive communication task in which every node locally broadcasts a packet to all its neighbors within a fixed distance. Assume that all communications proceed in synchronous time-slots and each node can transmit at most one fixed-size packet in each time-slot. The problem Minimumlatency beaconing schedule (MLBS) in multihop wireless networks seeks a shortest schedule for beaconing subject to the interference constraint. MLBS has been intensively studied since the mid-1980s, but all assume the protocol interference model with uniform interference radii. In this paper, we first present a constant-approximation algorithm for MLBS under the protocol interference model with arbitrary interference radii. Then, we develop a constant-approximation algorithm for MLBS under the physical interference model. Both approximation algorithms have efficient implementations in a greedy first-fit manner.

Index Terms-Beaconing schedule, protocol interference, physical interference, approximation algorithm
\end{abstract}

\section{INTRODUCTION}

Beaconing in multihop wireless networks is a communication task in which every node locally broadcasts a "beaconing" packet to all nodes within a certain distance referred to as the beaconing radius. It is frequently invoked by many networking protocols which require all nodes to collect the information of their nearby nodes. Thus, a fast beaconing is not only of theoretical interest, but also of practical importance. Assume that all communications proceed in synchronous time-slots and each node can transmit at most one fixed-size packet in each time-slot. A beaconing schedule assigns a time-slot to every node subject to the constraint that the nodes assigned in each time-slot are interference-free. The latency of a beaconing schedule is the number of time-slots during which at least one transmission occurs. The problem of computing a beaconing schedule with minimum latency in a wireless network is referred to as Minimum-Latency Beaconing Schedule (MLBS).

Variants of MLBS have been studied since the mid-1980s [2], [3], [4], [8], [9], [13], [11], [12], [14], [15]. All the prior works assumed either implicitly or explicitly the protocol interference model. An instance of a network is specified by a finite planar set $V$ of nodes. Each node $v$ has a unit beaconing

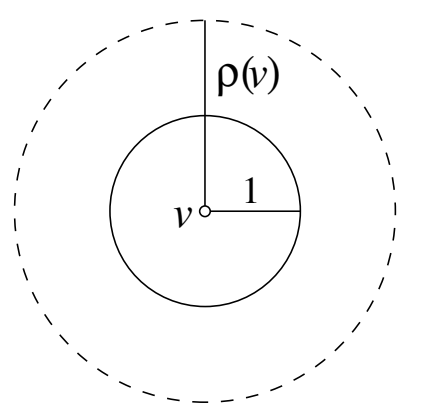

Fig. 1. The beaconing range and interfrence range of a node $v$.

radius, and its beaconing range is the unit disk centered at $v$. In addition, each node $v$ has an interference radius $\rho(v) \geq 1$ and its interference range is the disk centered at $v$ of radius $\rho(v)$ (see Figure 1). Then, a pair of nodes $u$ and $v$ cannot transmit in the same time-slot if and only if one of the three conditions holds: (1) $u$ and $v$ are within each other's beaconing range (see Figure 2(a)), (2) some node $w$ other than $u$ and $v$ is within $u$ 's beaconing range and $v$ 's interference range (see Figure 2(b)), and (3) some node $w$ other than $u$ and $v$ is within $v$ 's beaconing range and $u$ 's interference range (see Figure 2(c)). If $u$ and $v$ satisfy at least one these three conditions, they are said to conflict with each other; otherwise, they are said to be conflict-free or independent.

All prior works except [14] assumed implicitly that all nodes also have unit interference radius. In other words, each node has equal the interference radius and beaconing radius, both of which are equal to one. Sen and Huson [11] proved the NP-hardness of MLBS even in this simple setting. A classic greedy coloring algorithm due to [7] was adopted by Sen and Malesinska [12] to produce a beaconing schedule, whose approximation bound was shown to be 7 by Wan et al. [15] Recently, Wan et al. [14] considered a slightly more general scenario in which all nodes have uniform interference radius of arbitrary value $\rho \geq 1$. They proposed a different algorithm with approximation bound ranging from 3 to 6 depending on the value of $\rho$. In particular, when $\rho=1$ their algorithm 


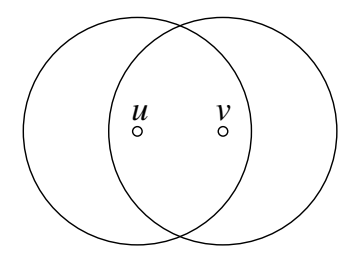

(a)

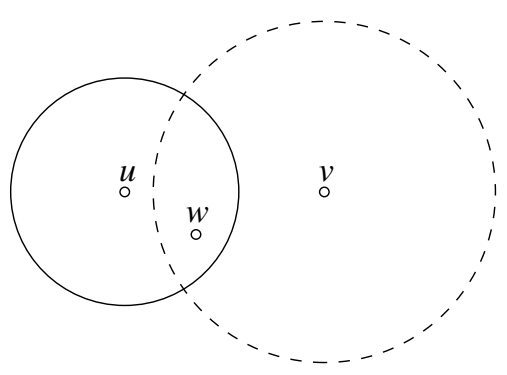

(b)

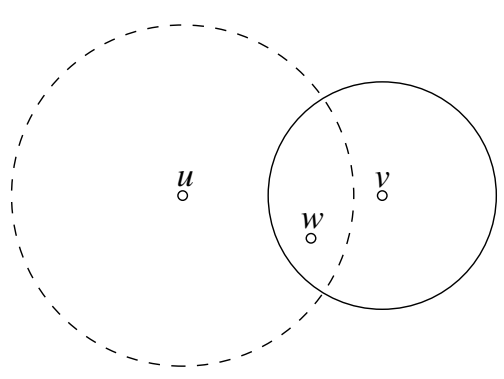

(c)

Fig. 2. Three possible scenarios of conflict between a pair of nodes $u$ and $v$ under the protocol interference model.

achieves an approximation bound 5 , which is better than the approximation bound 7 of the greedy algorithm proposed earlier.

This paper makes two contributions to the algorithmic studies of MLBS. Our first contribution is a constantapproximation algorithm for MLBS under the protocol interference model in which all nodes have arbitrary interference radii. Our second contribution is a constant-approximation algorithm for MLBS under the following physical interference model. All the networking nodes $V$ lie in a plane and transmit at a fixed power $P$. The path loss is then determined by a positive reference loss parameter $\eta$, and the path-loss exponent $\kappa$, which is a constant greater than 2 but less than 6 typically. Specifically, when a node $u$ transmits a signal at power $P$, the power of this signal captured by another node $v$ is $\eta P\|u v\|^{-\kappa}$, where $\|u v\|$ denotes the scaled Euclidean distance between $u$ and $v$ so that the the beaconing radius is one. The signal quality perceived by a receiver is measured by the signal to interference and noise ratio (SINR), which is the quotient between the power of the wanted signal and the total power of unwanted signals and the ambient-both internal and external-noise $\xi$. In order to correctly interpret the wanted signal, the SINR must be no less than certain threshold $\sigma$. Both of our approximation algorithms not only have constant approximation bounds, but also have efficient implementation in the greedy first-fit manner.

The remaining of this paper is organized as follows. In Section II, we introduce the first-fit coloring in general graphs and define a graph parameter useful in bounding approximation ratio of the first-fit coloring. In Section III, we present a firstfit beaconing schedule under the protocol interference model with arbitrary interference radii and analyze its approximation bound. In Section IV, we develop a first-fit beaconing schedule under the physical interference model and derive its approximation bound. Finally, we summarize this paper in Section V.

\section{FIRST-FIT COLORING}

Let $G=(V, E)$ be an undirected graph. The subgraph of $G$ induced by a subset $U$ of $V$ is denoted by $G[U]$. The minimum degree of $G$ is denoted by $\delta(G)$. A subset $I$ of $V$ is an independent set of $G$ if no two vertices in $I$ are adjacent. If $I$ is a independent set of $G$ but no proper superset of $I$ is a independent set of $G$, then $I$ is called a maximal independent set of $G$. A vertex coloring of $G$ is an assignment of colors to $V$ satisfying that adjacent vertices are assigned with distinct colors. Equivalently, a vertex coloring of $G$ is a partition of $V$ into independent sets. The chromatic number of $G$, denoted by $\chi(G)$, is the smallest number of colors required by any vertex coloring of $G$. The problem Minimum Vertex Coloring seeks a vertex coloring of a given graph with the fewest colors. It is NP-hard in general.

First-fit coloring is a simple greedy coloring algorithm. Given a vertex ordering $\left\langle v_{1}, v_{2}, \cdots, v_{n}\right\rangle$ of $V$, a coloring of $V$ with colors represented by natural numbers can be produced in the following first-fit manner: Assign the color 1 to $v_{1}$. For $i=2$ up to $n$, assign to $v_{i}$ with the smallest color which is not used by any neighbor of $v_{i}$ which precedes $v_{i}$. Such coloring of $V$ is referred to as the first-fit coloring in the ordering $\left\langle v_{1}, v_{2}, \cdots, v_{n}\right\rangle$. It is easy to verify that the number of colors used by the first-fit coloring in the ordering $\left\langle v_{1}, v_{2}, \cdots, v_{n}\right\rangle$ is at most $1+\max _{1<i \leq n}\left|V_{i}\right|$, where each $V_{i}$ with $1<i \leq n$ consists of all neighbors $v_{j}$ of $v_{i}$ with $1 \leq j<i$. The value $\max _{1<i \leq n}\left|V_{i}\right|$ is referred to as the inductivity of the ordering $\left\langle v_{1}, v_{2}, \cdots, v_{n}\right\rangle$.

Now, we describe a special vertex ordering, referred to as smallest-last ordering [7], which achieves the smallest inductivity. This ordering is produced iteratively as follows: Initialize $G^{\prime}$ to $G$. For $i=n$ down to 1 , let $v_{i}$ be a vertex of the smallest degree in $G^{\prime}$ and delete $v_{i}$ from $G^{\prime}$. Then, the 
ordering $\left\langle v_{1}, v_{2}, \cdots, v_{n}\right\rangle$ is a smallest-last ordering. It was proved in [7] among all vertex orderings., the smallest-last ordering achieves the smallest inductivity, which is given by

$$
\delta^{*}=\max _{U \subseteq V} \delta(G[U]) .
$$

The parameter $\delta^{*}$ is referred to as the inductivity of $G$.

Next, we introduce a graph parameter useful in bounding both the inductivity of a vertex ordering and the inductivity of $G$. Given a vertex ordering $\left\langle v_{1}, v_{2}, \cdots, v_{n}\right\rangle$ of $V$, its inductive local independence number (LIN) is defined to be the maximum size of any independent set contained in some $V_{i}$ with $1 \leq j<i$, where $V_{i}$ consists of all neighbors $v_{j}$ of $v_{i}$ with $1 \leq j<i$.

Lemma 1: For any vertex ordering $\left\langle v_{1}, v_{2}, \cdots, v_{n}\right\rangle$ of $G$, its inductivity is at most $\alpha^{*}(\chi(G)-1)$, where $\alpha^{*}$ is its inductive LIN.

Proof: For each $1 \leq i \leq n$, let $V_{i}$ denote the set of nodes $v_{j}$ with $1 \leq j<i$ adjacent to $v_{i}$. Then, at most $\alpha^{*}$ nodes in each $V_{i}$ can share the same color. Therefore, the nodes in $V_{i}$ require at least $\left\lceil\left|V_{i}\right| / \alpha^{*}\right\rceil$ colors. Since $v_{i}$ must be colored differently from its neighbors, $V_{i}$ and $v_{i}$ itself require at least $\left\lceil\left|V_{i}\right| / \alpha^{*}\right\rceil+1$ colors. Hence,

$$
\chi(G) \geq\left\lceil\left|V_{i}\right| / \alpha^{*}\right\rceil+1,
$$

which implies that

$$
\left|V_{i}\right| \leq \alpha^{*}(\chi(G)-1) .
$$

Thus, the inductivity of $\left\langle v_{1}, v_{2}, \cdots, v_{n}\right\rangle$ is at most $\alpha^{*}(\chi(G)-1)$.

From Lemma 1, we immediately get our first "master theorem" on the performance of a first-fit coloring.

Theorem 2: The first-fit coloring of $G$ in an ordering with inductive LIN $\alpha^{*}$ uses at most $\alpha^{*}(\chi(G)-1)+1$ colors, and hence has approximation bound $\alpha^{*}$.

From Lemma 1 and the fact that the smallest-last ordering has the smallest inductivity, we obtain our second "master theorem" on the performance of a first-fit coloring.

Theorem 3: Suppose that $G$ has a vertex ordering with inductive LIN $\alpha^{*}$. Then, the first-fit coloring in the smallest degree last ordering uses at most $\alpha^{*}(\chi(G)-1)+1$ colors, and hence has approximation bound $\alpha^{*}$.

\section{Beaconing Schedule Subject to Protocol INTERFERENCE}

Consider an instance of a multihop network under the protocol interference model specified by a finite planar set $V$ of nodes together with unit beaconing radius and an interference radius function $\rho \in \mathbb{R}_{+}^{V}$ satisfying that $\rho(v) \geq 1$ for each node $v \in V$. We define the conflict graph of $V$ as follows: For any pair of distinct nodes $u$ and $v$, there is an edge between $u$ and $v$ in the conflict graph of $V$ if and only if either one of the three conditions holds: (1) $\|u v\| \leq 1$, (2) there exists a node $w$ other than $u$ and $v$ satisfying that $\|u w\| \leq 1$ and $\|v w\| \leq \rho(v)$, (3) there exists a node $w$ other than $u$ and $v$ satisfying that $\|u w\| \leq \rho(u)$ and $\|v w\| \leq 1$. Then, a beaconing schedule for $V$ under the protocol interference model is equivalent to a coloring of $H$ with the latency corresponding to the number of colors. In particular, the beaconing schedule corresponding to the first-fit coloring of the conflict graph of $V$ in the smallestdegree-last ordering is referred to as the the first-fit beaconing schedule under the protocol interference model.

In this section, we prove the following approximation bound of the first-fit beaconing schedule under the protocol interference model.

Theorem 4: The approximation bound of the first-fit beaconing schedule under the protocol interference model is at most 61 .

To prove this theorem, we consider a node ordering called interference radius decreasing ordering, in which all nodes are sorted in descending order of their interference radii. We will show that its inductive LIN in the conflict graph is at most 61 , from which Theorem 4 follows immediately by Theorem 3 .

Lemma 5: The inductive LIN of interference radius decreasing ordering in the conflict graph is at most 61 .

The proof of this lemma utilizes the following classical result on circle packing due to Groemer [5].

Theorem 6 (Groemer Inequality): Suppose that $C$ is a compact convex set and $I$ is a set of points with mutual distances at least one. Then

$$
|I \cap C| \leq \frac{\operatorname{area}(C)}{\sqrt{3} / 2}+\frac{\operatorname{peri}(C)}{2}+1
$$

where area $(C)$ and peri $(C)$ are the area and perimeter of $C$ respectively.

When the set $C$ is a disk or a half-disk, we have the following packing bound. 
Corollary 7: Suppose that $S$ (respectively, $S^{\prime}$ ) is a disk (respectively, half-disk) of radius $r$, and $I$ is a set of points with mutual distances at least one. Then

$$
\begin{aligned}
& |I \cap S| \leq \frac{2 \pi r^{2}}{\sqrt{3}}+\pi r+1, \\
& \left|I \cap S^{\prime}\right| \leq \frac{\pi r^{2}}{\sqrt{3}}+\left(\frac{\pi}{2}+1\right) r+1 .
\end{aligned}
$$

In addition, the following geometric fact will be used in the proof of Lemma 5.

Lemma 8: Suppose that $\sqrt{7} \leq\|u v\| \leq\|u w\| \leq 1+$ $r$ and $\widehat{v u w} \leq \arccos \frac{5}{2 \sqrt{7}} \approx 19.107^{\circ}$. Then $\|v w\| \leq$ $\max \{1, r-1\}$.

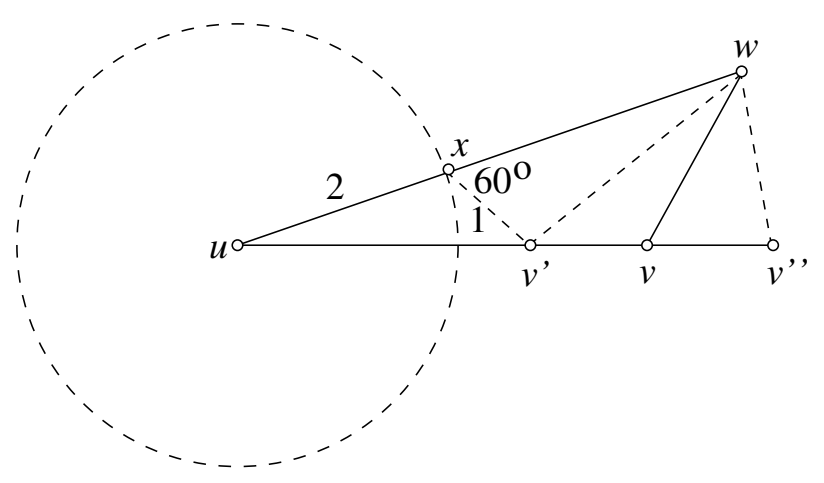

Fig. 3. $\|v w\| \leq \max \{1, r-1\}$.

Proof: Since $\|v w\|$ increases with $\widehat{v u w}$ while fixing the lengths of $u v$ and $u w$, it is sufficient to show that the lemma holds when $\widehat{v u w}=\arccos \frac{5}{2 \sqrt{7}}$. So, we assume that $\widehat{v u w}=$ $\arccos \frac{5}{2 \sqrt{7}}$. Let $v^{\prime}$ be the point in the ray $u v$ satisfying that $\left\|u v^{\prime}\right\|=\sqrt{7}$, and $v^{\prime \prime}$ be the point in the ray $u v$ satisfying that $\left\|u v^{\prime \prime}\right\|=\|u w\|$ (see Figure 3). The $v$ lies on the segment $v^{\prime} v^{\prime \prime}$. It's sufficient to show that both $\left\|v^{\prime} w\right\|$ and $\left\|v^{\prime \prime} w\right\|$ are at most $\max \{1, r-1\}$. Let $x$ be the intersection point on $u w$ with $\|u x\|=2$. Then,

$$
\|w x\|=\|u w\|-2 \leq 1+r-2=r-1 .
$$

Since $\left\|u v^{\prime}\right\|=\sqrt{7}$, we have $\left\|v^{\prime} x\right\|=1$ and $\widehat{u y v^{\prime}}=120^{\circ}$. Hence $\widehat{v^{\prime} x w}=60^{\circ}$. Hence,

$$
\left\|v^{\prime} w\right\|<\max \left\{\left\|v^{\prime} x\right\|,\|w x\|\right\} \leq \max \{1, r-1\} .
$$

On the other hand,

$$
\begin{aligned}
\left\|v^{\prime \prime} w\right\| & =2\|u w\| \sin \frac{\widehat{v u w}}{2}<\frac{\|u w\|}{3} \\
& \leq \frac{1+r}{3} \leq \max \{1, r-1\} .
\end{aligned}
$$

So, the lemma follows.

Now, we are ready to prove Lemma 5. Consider an arbitrary node $u$ in the interference radius decreasing ordering. Let
$I$ be an independent set of nodes which are neighbors of $u$ in the conflict graph and precede $u$ in the interference radius decreasing ordering. We partition $I$ into two subsets. $I_{1}$ consists of the nodes in $I$ whose distances from $u$ are at most $\max \{\sqrt{7}, \rho(u)+1\}$, and $I_{2}=I \backslash I_{1}$ (see Figure 4). Clearly, all nodes in $I_{1}$ have mutual distances greater than $\max \{1, \rho(u)-1\}$. To bound $\left|I_{1}\right|$, we show that

$$
\frac{\max \{\sqrt{7}, \rho(u)+1\}}{\max \{1, \rho(u)-1\}} \leq 3 .
$$

If $1 \leq \rho(u) \leq \sqrt{7}-1$, then

$$
\frac{\max \{\sqrt{7}, \rho(u)+1\}}{\max \{1, \rho(u)-1\}}=\sqrt{7} \leq 3 .
$$

If $\sqrt{7}-1<\rho(u) \leq 2$, then

$$
\frac{\max \{\sqrt{7}, \rho(u)+1\}}{\max \{1, \rho(u)-1\}}=\rho(u)+1 \leq 3 .
$$

If $\rho(u)>2$, then

$$
\begin{aligned}
& \frac{\max \{\sqrt{7}, \rho(u)+1\}}{\max \{1, \rho(u)-1\}}=\frac{\rho(u)+1}{\rho(u)-1} \\
& =1+\frac{2}{\rho(u)-1}<1+\frac{2}{2-1}=3 .
\end{aligned}
$$

Therefore,

$$
\frac{\max \{\sqrt{7}, \rho(u)+1\}}{\max \{1, \rho(u)-1\}} \leq 3 .
$$

By Corollary 7,

$$
\left|I_{1}\right| \leq\left\lfloor\frac{2 \pi}{\sqrt{3}} \cdot 3^{2}+3 \pi+1\right\rfloor=43 .
$$

Note that all nodes in $I_{2}$ are in-neighbors of $u$ in the interference digraph. By Lemma 8, any pair of nodes in $I_{2}$ are separated by an angle greater than $\arccos \frac{5}{2 \sqrt{7}}$. Hence,

$$
\left|I_{2}\right| \leq\left\lceil\frac{2 \pi}{\arccos \frac{5}{2 \sqrt{7}}}\right\rceil-1=18
$$

Therefore,

$$
|I|=\left|I_{2}\right|+\left|I_{2}\right| \leq 43+18=61 .
$$

So, Lemma 5 holds.

In the remaining of this section, we will derive the approximation bound in case of all nodes have uniform interference radii equal to $\rho$. For any $r \geq 1$, let $\mu(r)$ denote the maximum number of points in a half-disk of radius $r$ whose mutual distances are greater than one. By Corollary 7, we have

$$
\mu(r) \leq\left\lfloor\frac{\pi r^{2}}{\sqrt{3}}+\left(\frac{\pi}{2}+1\right) r\right\rfloor+1 .
$$

Theorem 9: In case of uniform interference radii $\rho$, the approximation bound of the first-fit beaconing scheduling is at most $\mu\left(\frac{\rho+1}{\max \{1, \rho-1\}}\right)$ in general, and at most 7 when $\rho=1$. 


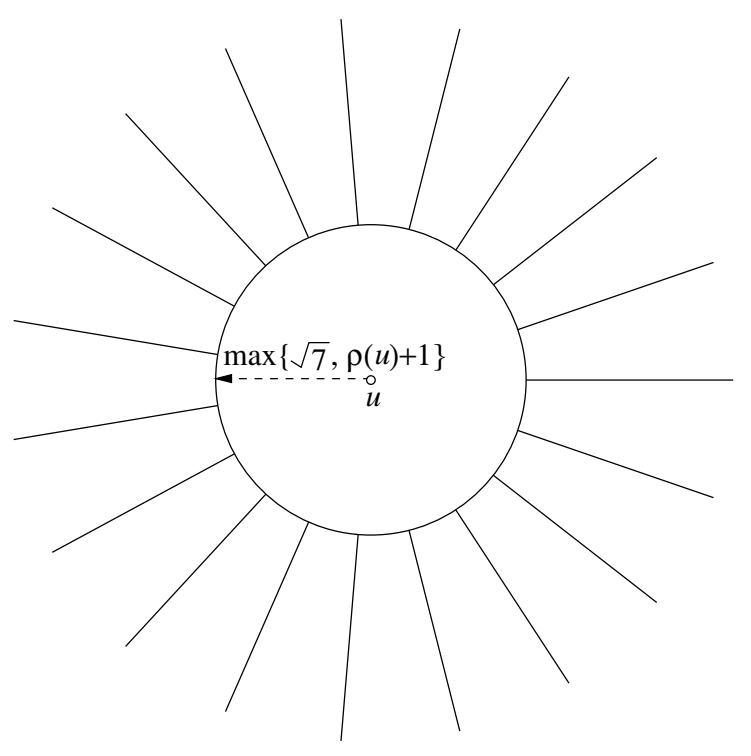

Fig. 4. $I_{1}$ consists of all nodes inside the disk, $I_{2}$ consists of all nodes outside the disk.

Proof: The approximation bound 7 when $\rho=1$ was proved in [15]. So we assume that $\rho>1$. Consider the lexicographic ordering of $V$, in which all nodes are sorted in the lexicographic order of their positions. We prove that its inductive LIN is at most $\mu\left(\frac{\rho+1}{\max \{1, \rho-1\}}\right)$ in the conflict graph. Consider an arbitrary node $v$. Let $U$ denote all the neighbors of $v$ in the conflict graph which precedes $v$ in the lexicographic ordering. Then, all nodes in $U$ lie in the left half-disk of radius $\rho+1$ centered at $v$. Let $I$ be an independent set of the conflict graph contained in $U$. Then, the mutual distances of the nodes in $I$ are greater than $\min \{1, \rho-1\}$. By Corollary 7 ,

$$
|I| \leq \mu\left(\frac{\rho+1}{\max \{1, \rho-1\}}\right) .
$$

Therefore, the inductive LIN of the lexicographic ordering in the conflict graph is at most $\mu\left(\frac{\rho+1}{\max \{1, \rho-1\}}\right)$. By Theorem 3, the theorem follows.

Note that

$$
\begin{aligned}
& \mu\left(\frac{\rho+1}{\max \{1, \rho-1\}}\right) \\
& \leq\left\lfloor\frac{\pi\left(\frac{\rho+1}{\max \{1, \rho-1\}}\right)^{2}}{\sqrt{3}}+\left(\frac{\pi}{2}+1\right) \frac{\rho+1}{\max \{1, \rho-1\}}\right\rfloor+1 .
\end{aligned}
$$

Figure 5 is a plot of such upper bound on $\mu\left(\frac{\rho+1}{\max \{1, \rho-1\}}\right)$. As shown in the figure, this upper bound achieves the maximum 25 at $\rho=2$.

In summary, the first-fit beaconing schedule under protocol interference model is a 61-approximation in general, a $\mu\left(\frac{\rho+1}{\max \{1, \rho-1\}}\right)$-approximation when all nodes have uniform

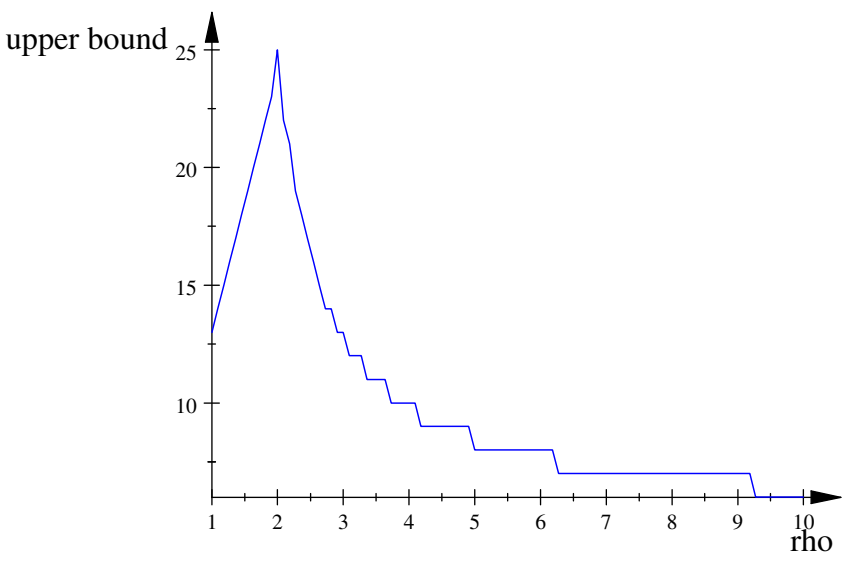

Fig. 5. The plot of an upper bound on $\mu\left(\frac{\rho+1}{\max \{1, \rho-1\}}\right)$.

interference radius $\rho$, and a 7-approximation when all nodes have unit interference radii.

\section{Beaconing Schedule Subject to Physical INTERFERENCE}

Consider an instance of a multihop network under the protocol interference model specified by a finite planar set $V$ of nodes together with their transmission power $P$. By proper scaling, we assume that the beaconing radius is one. When a node $u$ transmits a signal at power $P$, the power of this signal captured by another node $v$ is $\eta P\|u v\|^{-\kappa}$, where $\eta$ is the reference loss parameter, and $\kappa$ is the path-loss exponent greater than 2 but less than 6 typically. Let $\xi$ be the noise power. In the presence of the interference from a set $W$ of nodes, the SINR of the signal from a node $u \notin W$ at a node $v \notin W$ is given by

$$
\frac{\eta P\|u v\|^{-\kappa}}{\xi+\sum_{w \in W} \eta P\|w v\|^{-\kappa}},
$$

and $v$ can successfully receive the signal from $u$ if and only in the above SINR is no less than a threshold $\sigma$. Let

$$
R=\left(\frac{\eta P}{\sigma \xi}\right)^{1 / \kappa}
$$

Then, a pair of nodes $u$ and $v$ can communicate with each other in the absence of interference if and only if $\|u v\| \leq R$. The value $R$ is thus referred to as the maximum transmission radius. Let $\lambda=1 / R$, i.e. the ratio of the beaconing radius and the maximum transmission radius. We assume $\lambda \leq 1-\varepsilon$ for some small positive constant $\varepsilon$. This assumption is valid in almost all practical applications.

A set $I$ of nodes is said to be independent under the physical interference model if (1) the mutual distances of the nodes in $I$ are greater than one, and (2) when all nodes in $I$ transmit 
simultaneously, the transmission by each node $u \in I$ can be received successfully by all nodes within a unit distance from $u$. Then, any beaconing schedule is a partition of $V$ into independent sets. We first present a sufficient condition for a set of nodes to be independent. Our condition makes use of the Riemann zeta function in the following form :

$$
\zeta(x)=\sum_{j=1}^{\infty} \frac{1}{j^{x}} .
$$

Note $\zeta(1)=\infty$, and for any $x>1$,

$$
\zeta(x)=\sum_{j=1}^{\infty} \frac{1}{j^{x}} \leq 1+\int_{1}^{\infty} \frac{1}{y^{x}} d y=\frac{x}{x-1} .
$$

The values of $\zeta(x)$ for small values of $x$ are

$$
\begin{aligned}
\zeta(1.5) & =2.612, \\
\zeta(2) & =\frac{\pi^{2}}{6}=1.6449, \\
\zeta(2.5) & =1.341 \\
\zeta(3) & =1.202 \\
\zeta(3.5) & =1.127 \\
\zeta(4) & =\frac{\pi^{4}}{90}=1.082, \\
\zeta(5) & =1.041 \\
\zeta(6) & =\frac{\pi^{6}}{945}=1.017 .
\end{aligned}
$$

Define

$$
\rho=1+\left(\frac{\sigma(16 \zeta(\kappa-1)+8 \zeta(\kappa)-6)}{1-\lambda^{\kappa}}\right)^{1 / \kappa} .
$$

Then, we have the following simple sufficient condition for a set $I$ to be independent.

Lemma 10: Suppose that $I$ is a set of nodes whose mutual distances are greater than $\rho$. Then, $I$ is an independent set under the physical interference model.

Proof: Consider a node $u \in I$ and a node $v$ with $0<$ $\|u v\| \leq 1$. Clearly, $v \notin I$. We first show that

$$
\sum_{w \in I \backslash\{u\}}\left(\frac{\|u v\|}{\|w v\|}\right)^{\kappa}<\frac{1-\lambda^{\kappa}}{\sigma} .
$$

For each $j \geq 1$, define

$$
I_{j}=\{w \in I: j \rho \leq\|u w\|<(j+1) \rho\},
$$

Then, $I_{1}, I_{2}, \cdots$ form a partition of $I \backslash\{u\}$. By a classic result due to Bateman and Erdös [1], $\left|I_{1}\right| \leq 18$. For $j \geq 2$, using the folklore area argument we have

$$
\begin{aligned}
\left|I_{j}\right| & \leq \frac{\pi\left((j+1) \rho+\frac{\rho}{2}\right)^{2}-\pi\left(j \rho-\frac{\rho}{2}\right)^{2}}{\pi\left(\frac{\rho}{2}\right)^{2}} \\
& \leq 8(2 j+1) .
\end{aligned}
$$

Consider a node $v$ in the unit-disk centered at $u$. Then, for each $w \in I_{j}$ with $j \geq 1$,

$$
\|w v\| \geq\|w u\|-\|u v\| \geq j \rho-1 \geq j(\rho-1) .
$$

So,

$$
\begin{aligned}
& \sum_{w \in I \backslash\{u\}}\left(\frac{\|u v\|}{\|w v\|}\right)^{\kappa} \\
= & \sum_{j=1}^{\infty} \sum_{w \in I_{j}}\left(\frac{\|u v\|}{\|w v\|}\right)^{\kappa} \\
< & \sum_{j=1}^{\infty} \frac{\left|I_{j}\right|}{(j(\rho-1))^{\kappa}} \\
= & (\rho-1)^{-\kappa}\left(\left|I_{1}\right|+\sum_{j=2}^{\infty} \frac{\left|I_{j}\right|}{j^{\kappa}}\right) \\
< & (\rho-1)^{-\kappa}\left(18+8 \sum_{j=2}^{\infty} \frac{2 j+1}{j^{\kappa}}\right) \\
\leq & (\rho-1)^{-\kappa}(16 \zeta(\kappa-1)+8 \zeta(\kappa)-6) \\
= & \frac{1-\lambda^{\kappa}}{\sigma} .
\end{aligned}
$$

Next, we show that the SINR of the signal from $u$ at $v$ exceeds $\sigma$. In the presence of the interference from $I \backslash\{u\}$, the SINR of the signal from $u$ at $v$ is

$$
\begin{aligned}
& \frac{\eta P\|u v\|^{-\kappa}}{\xi+\sum_{w \in I \backslash\{u\}} \eta P\|w v\|^{-\kappa}} \\
& =\frac{1}{\frac{\xi}{\eta P}\|u v\|^{\kappa}+\sum_{w \in I \backslash\{u\}}\left(\frac{\|v v\|}{\|w v\|}\right)^{\kappa}} \\
& =\frac{1}{\frac{1}{\sigma}\left(\frac{\|u v\|}{R}\right)^{\kappa}+\sum_{w \in I \backslash\{u\}}\left(\frac{\|u v\|}{\|w v\|}\right)^{\kappa}} \\
& >\frac{1}{\frac{\lambda^{\kappa}}{\sigma}+\frac{1-\lambda^{\kappa}}{\sigma}} \\
& =\sigma .
\end{aligned}
$$

So, the lemma follows.

We remark that for practical applications, the value of $\rho$ is a small constant. Figure 6 is a plot of an upper bound on $\rho$ for $\kappa=4$ and $\sigma=16$. When $\lambda=0.99,0.95,0.9,0.81, \rho$ is at most $10.71,7.6,6.6 .5,6$ respectively.

For any $r>0$, the $r$-disk graph on $V$ is an undirected graph in which there is an edge between two nodes $u$ and $v$ if and only if $0<\|u v\| \leq r$. Then, a set $I$ of nodes is an independent set of the $r$-disk graph on $V$ if and only if their mutual distances are greater than $r$. So, Lemma 10 has the following alternative interpretation: Any independent set of the $\rho$-disk graph on $V$ is an independent set under the physical interference model. As a result, any coloring of the 


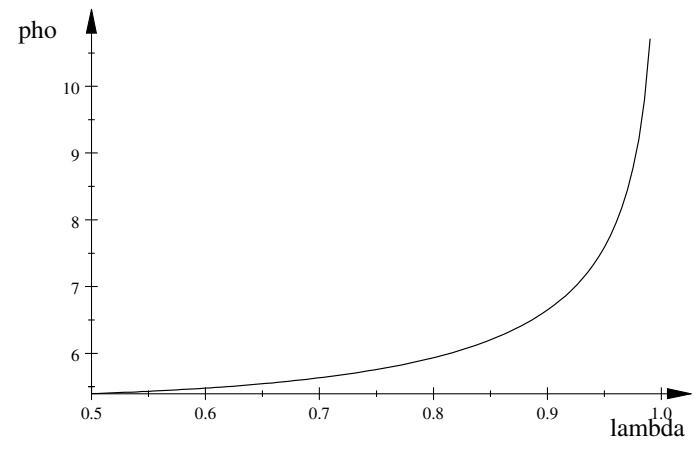

Fig. 6. An upper bound on $\rho$ when $\kappa=4$ and $\sigma=16$.

$\rho$-disk graph on $V$ gives rise to a beaconing schedule under the physical interference model. In particular, the beaconing schedule corresponding to the first-fit coloring of the $\rho$-disk graph on $V$ in the smallest-degree-last ordering is referred to as the the first-fit beaconing schedule under the physical interference model.

In the remaining of this section, we prove the following approximation bound of the first-fit beaconing schedule under the physical interference model.

Theorem 11: The approximation bound of the first-fit beaconing schedule under the physical interference model is at most $\mu(\rho)$.

Proof: Let opt be the latency of a shortest beaconing schedule under the physical interference model. We first prove that the inductivity of the lexicographic ordering in the $\rho$-disk graph on $V$ is at most $\mu(\rho) \cdot o p t-1$. Consider an arbitrary node $v$. Let $U$ denote all the neighbors of $v$ in the $\rho$-disk graph on $V$ which precedes $v$ in the lexicographic ordering. Then, all nodes in $U$ lie in the left half-disk of radius $\rho$ centered at $v$. Then, at most $\mu(\rho)$ nodes in $U \cup\{u\}$ have mutual distances greater than one. On the other hand, in any beaconing schedule, all the nodes transmitting in a time-slot must have mutual distances greater than one. Therefore, in any time-slot of any shortest beaconing schedule under the physical interference model, at most $\mu(\rho)$ nodes come from $U \cup\{u\}$. So,

$$
\text { opt } \cdot \mu(\rho) \geq|U \cup\{u\}|=|U|+1,
$$

which implies that

$$
|U| \leq \mu(\rho) \cdot o p t-1 .
$$

Therefore, the inductivity of the lexicographic ordering in the $\rho$-disk graph on $V$ is at most $\mu(\rho) \cdot$ opt -1 .

Since the smallest-last ordering has the smallest inductivity, its inductivity in the $\rho$-disk graph on $V$ is also at most $\mu(\rho)$. opt -1 . Thus, the first-fit coloring of the $\rho$-disk graph on $V$ in the smallest-last ordering uses at most

$$
\mu(\rho) \cdot \text { opt }-1+1=\mu(\rho) \cdot \text { opt }
$$

colors. This means that the latency of the first-fit beaconing schedule under the physical interference model is at most $\mu(\rho) \cdot$ opt. Thus, the theorem holds.

\section{CONCLUSION}

MLBS under the protocol interference model with uniform interference radii has been well studied in the literature. However, MLBS under the protocol interference model with arbitrary interference radii and MLBS under the physical interference model have not been explored before. In this paper, we first developed a first-fit beaconing schedule under the protocol interference model with arbitrary interference radii. This schedule is a 61-approximation in general, a 25-approximation when all nodes have uniform interference radius, and a 7approximation when all nodes have unit interference radii. Then, we developed another first-fit beaconing schedule under the physical interference model. Its approximation ratio is bounded by a geometric packing parameter $\mu(\rho)$, where $\rho$ is a small constant depending on the ratio of the beaconing radius to the maximum transmission radius, and $\mu(\rho)$ is the maximum number of points in a half-disk of radius $\rho$ with mutual distances greater than one. Both first-fit beaconing schedules can be computed efficiently in a greedy first-fit manner.

\section{ACKNOWLEDGMENT}

Peng-Jun Wan, Zhu Wang, and Hongwei Du were supported in part by National Science Foundation of USA under grants CNS-0831831 and CNS-0916666. Scott C.-H. Huang and Zhiyuan Wan were supported in part by the Research Grants Council of Hong Kong under the project GRF CityU 113807.

\section{REFERENCES}

[1] P. Bateman and P. Erdös, Geometrical extrema suggested by a lemma of besicovitch, The American Mathematical Monthly, pp. 306-314, May 1951.

[2] I. Chlamtac and A. Farago, Making Transmission Schedules Immune to Topology Changes in Multi-Hop Packet Radio Networks, IEEE/ACM Transactions on Networking, Vol. 2, No. 1, pp. 23-29, Feb. 1994.

[3] I. Chlamtac and S. Kutten, A spatial reuse tdma/fdma for mobile multihop radio nertworks, in IEEE INFOCOM, pp. 389-394, March 1985.

[4] A. Ephremides and T.V. Truong, Scheduling Broadcasts in Multihop Radio Networks, IEEE Transactions on Communications, vol. 38, no. 4, pp. 456-60, April 1990.

[5] H. Groemer, Über die Einlagerung von Kreisen in einen konvexen Bereich. Math. Z., 73:285-294, 1960.

[6] P. Gupta and P.Kumar, The capacity of wireless networks, IEEE Trans. Inform. Theory, vol. 46, pp. 388-404, Mar. 2000. 
[7] D. W. Matula and L. L. Beck. Smallest-last ordering and clustering and graph coloring algorithms, Journal of the Association of Computing Machinery, 30(3):417-427, 1983.

[8] S. Ramanathan and E.L. Lloyd, Scheduling algorithms for multi-hop radio networks, IEEE/ACM Transactions on Networking, vol. 1, pp. 166172, April 1993.

[9] R. Nelson and L. Kleinrock, Spatial-TDMA: A collision-free multihop channel access protocol, IEEE Transactions on Communications, vol. 33, no. 9, pp. 934944, Sep. 1985.

[10] I. Niven, H.S. Zuckerman, and H.L. Montgomery, An introduction to the theory of numbers. Wiley, 5th ed., 1991.

[11] A. Sen and M. L. Huson, A New Model for Scheduling Packet Radio Networks, ACM/Baltzer Journal Wireless Networks, 3 (1997), pp. 71-82.
[12] A. Sen and E. Malesinska, Approximation Algorithms for Radio Network Scheduling, Proceedings of 35th Allerton Coneference on Communication, Control and Computing, Champaign, Illinois, pp. 573-582, October, 1997.

[13] D.S. Stevens and M.H. Ammar, Evaluation of slot allocation strategies for TDMA protocols in packet radio networks, IEEE Military Communications Conference, pp: 835-839, 1990.

[14] P.-J. Wan, X. Xu, L. Wang, X. Jia, and E.K. Park, Minimum-Latency Beaconing Schedule in Multihop Wireless Networks, IEEE INFOCOM 2009.

[15] P.-J. Wan, C.-W. Yi, X. Jia, and D. Kim: Approximation Algorithms for Conflict-Free Channel Assignment in Wireless Ad Hoc Networks, Wiley Journal on Wireless Communications and Mobile Computing, 6(2):201211, March 2006. 